\title{
Resolution of acute lung injury and inflammation: a translational mouse model
}

\author{
Brijesh V. Patel, Michael R. Wilson and Masao Takata
}

ABSTRACT: Previous animal models of acute lung injury (ALI) are limited as they only reproduce part of the complex pathobiology of clinical ALI. Here we develop a translational mouse model of $A L I$, which not only reflects the major clinical and pathological features but also enables investigation into ALI resolution.

Anaesthetised mice underwent orotracheal instillation of hydrochloric acid. During the immediate period after instillation, mice were carefully maintained with supplemental oxygen to avoid mortality. At specified time-points, lung injury was assessed by analysis of blood gases, respiratory mechanics, bronchoalveolar lavage fluid, alveolar fluid clearance and lung histology.

Animals exhibited significant weight loss, decreased oxygenation, increased respiratory elastance and pulmonary inflammation (intra-alveolar leukocyte influx/cytokine levels and histological injury scores). Moreover, mice displayed alveolar-capillary barrier dysfunction/ epithelial injury as reflected by increased alveolar protein, lung wet/dry weight ratio and soluble receptor for advanced glycation end-products, as well as reduced alveolar fluid clearance. These injury parameters peaked between days 1 and 3, followed by almost complete recovery over days 5-10. Histology showed evidence of fibrosis on day 10.

The results indicate that this resolving model of acid aspiration represents a powerful experimental tool to investigate the injurious, inflammatory, fibrotic, and resolving and reparative processes of ALI.

KEYWORDS: Acid aspiration, acute respiratory distress syndrome, alveolar epithelium, alveolar fluid clearance, inflammation, pulmonary oedema

$\mathbf{A}$ cute lung injury (ALI), or its more severe form acute respiratory distress syndrome (ARDS), continues to cause significant morbidity and mortality [1]. It is characterised by an acute exudative phase involving pulmonary oedema and inflammation, which lead to severe hypoxaemia, often necessitating mechanical ventilation. Recovery from ALI involves phases of resolution and repair, which may include substantial lung fibrosis. However, the mechanisms that govern whether and how these pathophysiological processes occur remain poorly understood [2]. One of the primary reasons for this lack of understanding is the nature of existing pre-clinical models, insofar as none fully replicates the complex pathobiology of clinical ALI. The majority of animal studies have focused on mechanisms within the acute exudative phase, and very few models allow examination of the later phases of inflammation, resolution and repair. These issues have been particularly highlighted in a recent American Thoracic Society workshop report on the features required to model experimental ALI in animals [3], as well as by other reviews highlighting the need for true translational modelling of ALI/ARDS $[4,5]$.

The acid aspiration paradigm has been described as potentially the most translatable model of direct ALI/ARDS, because there is a clear clinical correlate [5]. Despite this, only 3\% of recent literature indicates having utilised acid aspiration [4]. This is most likely to be due to a narrow dosing window between no injury and overwhelming injury leading to high mortality. To circumvent this issue, most models utilising acid aspiration either include mechanical ventilation to maintain animals, which limits investigators to the acute phases $(<4-6 \mathrm{~h})$ of ALI/ARDS [6, 7], or use lower acid volume, higher $\mathrm{pH}$ and/or unilateral pulmonary instillation to improve survival $[8,9]$. However, these latter approaches arguably may not reproduce the severity (not to
AFFILIATIONS

Section of Anaesthetics, Pain

Medicine and Intensive Care, Faculty of Medicine, Imperial College London, Chelsea and Westminster Hospital, London, UK.

\section{CORRESPONDENCE}

M. Takata

Anaesthetics, Pain Medicine and

Intensive Care

Imperial College London

Chelsea and Westminster Hospital

369 Fulham Road

London SW10 9NH

UK

E-mail:m.takata@imperial.ac.uk

Received:

June 022011

Accepted after revision:

Sept 232011

First published online:

Oct 172011 
mention bilateral nature) of clinical ALI that is required to be truly translational.

In this study, we have developed, optimised and characterised a model of acid aspiration that reproduces many of the physiological, biological and pathological changes found in human ALI/ ARDS, and, moreover, shows virtually full resolution by 510 days. We believe that this model will be of substantial use to further our understanding of human ALI/ARDS, in particular those processes regulating resolution and repair.

\section{METHODS}

\section{Animal model of acid aspiration}

All protocols were approved by the Ethical Review Board of Imperial College London (London, UK), and carried out under the authority of the UK Home Office in accordance with the Animals (Scientific Procedures) Act 1986, UK. We used male C57BL/ 6 mice (Charles River, Margate, UK) aged 10-12 weeks and weighing 25-30 g. In total, 68 animals were used: 23 for measurements of respiratory mechanics and alveolar inflammation, 25 for assessing alveolar fluid clearance, and 20 for lung wet/dry weight and histology scoring.

Mice were anaesthetised by intraperitoneal injection of xylazine $\left(6 \mathrm{mg} \cdot \mathrm{kg}^{-1}\right)$ and ketamine $\left(60 \mathrm{mg} \cdot \mathrm{kg}^{-1}\right)$, and given an intraperitoneal fluid bolus of $10 \mu \mathrm{L} \cdot \mathrm{g}^{-1} 0.9 \%$ normal saline as pre-emptive fluid resuscitation. Mice were suspended vertically from their incisors on a custom-made mount for orotracheal instillation, as described previously [10] (additional details are provided in the online supplementary material). A fine catheter was guided $1 \mathrm{~cm}$ below the vocal cords, and $75 \mu \mathrm{L}$ of an iso-osmolar (to mouse plasma, i.e. $322 \mathrm{mOsm} \cdot \mathrm{L}^{-1}$ ) solution of $0.1 \mathrm{M}$ hydrochloric acid ( $\mathrm{pH}$ 1.0) was instilled. For the next $4 \mathrm{~h}$, during which time animals exhibited significant respiratory depression/distress as an acute result of acid aspiration-induced ALI, mice were kept in a custommade transparent recovery box under humidified supplemental oxygen (inspiratory oxygen fraction $\left(\mathrm{FI}_{1} \mathrm{O}_{2}\right)$ reduced gradually from 1.0 to 0.21$)$. During this period, animals were carefully monitored and body temperature was maintained using external heat sources, after which they were transferred to individually ventilated cages with air and free access to food and water.

\section{Physiological measurements}

At specified time-points (1, 2, 3, 5 and 10 days) after acid instillation, animals were weighed and underwent surgical preparation for mechanical ventilation, to conduct standardised assessment of pulmonary gas exchange capability and respiratory mechanics. The preparation for mouse mechanical ventilation and pulmonary function tests has been described in detail previously [11, 12]. In brief, mice were anaesthetised, tracheostomised and ventilated using a custom-made ventilator, with the left carotid artery cannulated for monitoring arterial blood gases and blood pressure. After an initial lung recruitment manoeuvre $\left(30 \mathrm{cmH}_{2} \mathrm{O}\right.$ for $5 \mathrm{~s}$ ), animals were ventilated for $30 \mathrm{~min}$ (tidal volume $8-9 \mathrm{~mL} \cdot \mathrm{kg}^{-1}$, positive end-expiratory pressure $2.5 \mathrm{cmH}_{2} \mathrm{O}$, respiratory rate 120 breaths $\cdot \mathrm{min}^{-1}$ and $\mathrm{FI}, \mathrm{O}_{2} 1.0$ ) to standardise the volume history of the lungs. At the end of ventilation, blood gases were measured and respiratory mechanics determined using the end-inspiratory occlusion technique, as described previously $[11,13]$. Acid-injured animals were compared with otherwise untreated mice, receiving just the surgical preparation and 30-min ventilation procedures.

\section{Pulmonary inflammation}

At the end of the above procedure, mice were sacrificed by anaesthetic overdose. Bronchoalveolar lavage (BAL) was performed with $750 \mu \mathrm{L}$ of saline as described previously [11] and the samples were centrifuged at $240 \times g$. Protein levels in BAL fluid (BALF) were quantified (Bio-Rad Laboratories, Hemel Hempstead, UK) and BALF levels of interleukin (IL)-6, tumour necrosis factor (TNF)- $\alpha$, keratinocyte-derived chemokine (KC), macrophage inflammatory protein (MIP)-2 and soluble receptor for advanced glycation end-products (RAGE) were determined using ELISA kits (R\&D Systems, Abingdon, UK). BALF cell counts were obtained using a haemocytometer, with differential cytology performed on DiffQuik-stained samples prepared by cytospin (Shandon, Runcorn, UK).

\section{Alveolar fluid clearance}

In a separate series of experiments, alveolar fluid clearance (AFC) was determined at days 1, 2, 3, 5 and 10 after acid instillation (and in untreated animals), using a modification of previously described in situ models [14]. Mice were anaesthetised, tracheostomised, cannulated via the carotid artery and ventilated as described above. After intravenous heparinisation (20 units), animals were sacrificed and exsanguinated to drain pulmonary vessels and minimise any potential impact of pulmonary vascular pressure on AFC. Each animal then received three lung recruitment manoeuvres to standardise volume history of the lungs and further empty compliant vessels. The entire procedure took $<15 \mathrm{~min}$.

Mice then underwent intratracheal instillation with $700 \mu \mathrm{L}$ of an iso-osmolar medium containing 5\% low endotoxin bovine serum albumin (BSA; Sigma-Aldrich Ltd, Gillingham, UK) and $50 \mu \mathrm{g} \cdot \mathrm{mL}^{-1}$ fluorescent AlexaFluor 594-conjugated BSA (Invitrogen, Paisley, UK). Without disconnecting the instillation syringe, $400 \mu \mathrm{L}$ of instillate was immediately withdrawn and re-instilled, followed by removal of a first aliquot of $200 \mu \mathrm{L}$ as a time (t) zero reference sample. This process ensured homogeneous distribution of the instillate and that the reference sample was as representative of alveolar contents as possible. Mice were placed on a continuous positive airway pressure system delivering $100 \%$ oxygen at $8 \mathrm{cmH}_{2} \mathrm{O}$, and maintained at a temperature of $36.5-38^{\circ} \mathrm{C}$ throughout. $30 \mathrm{~min}$ after instillation, a surgical pneumothorax was induced to maximise recovery of the remaining instillate from the lungs. Percentage AFC over 30 min was determined by the equation:

$$
\left[1-\left(F_{0} / F_{30}\right)\right] \times 100
$$

where $F_{0}$ is the fluorescence of the $t=0$ min reference sample and $F_{30}$ is the fluorescence of the $t=30 \mathrm{~min}$ sample. More detailed methodology can be found in the online supplementary material.

\section{Lung injury scoring}

In a separate series of experiments, acid injured mice (at each time-point) and untreated animals were sacrificed and their left lungs instilled and inflated at $15 \mathrm{cmH}_{2} \mathrm{O}$ with $4 \%$ paraformaldehyde and removed for paraffin embedding. Slices at $10-\mu \mathrm{m}$ thickness were subsequently stained with haematoxylin and eosin (Sigma-Aldrich Ltd), or Masson's trichrome stain (Sigma-Aldrich Ltd) for fibrosis. Lung injury scores were quantified by an investigator blinded to the 

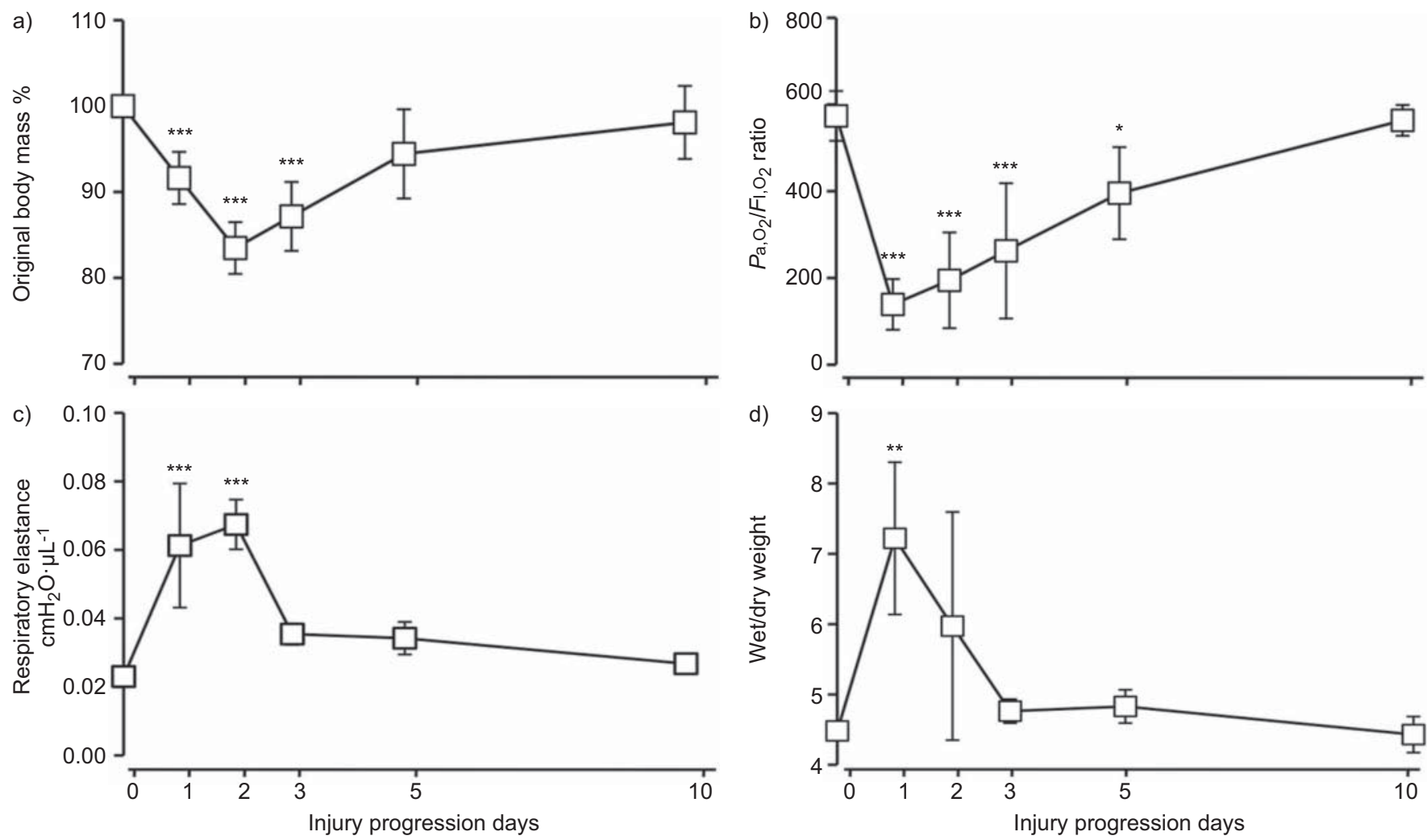

FIGURE 1. a) Body weight changes as represented by percentage of original body mass ( $n=9-11$ for each time-point). b) Arterial oxygenation as measured by arterial oxygen tension $\left(\mathrm{Pa}_{1} \mathrm{O}_{2}\right)$ /inspiratory oxygen fraction $\left(\mathrm{F}, \mathrm{O}_{2}\right)$ at different stages of lung injury $(\mathrm{n}=5-7$ for each time-point). c) Respiratory system elastance ( $\mathrm{n}=3-5$ for each time-point) and d) lung wet/dry weight ratio ( $n=3-4$ for each time-point) at different time-points after acid instillation. *: $p<0.05 ; * *: p<0.01$; ***: $p<0.001$ versus day 0 uninjured controls.

treatment groups using recently published criteria, which gives an overall score of between 0 and 1 (see online supplementary material for details) [3]. The right lungs were tied off prior to paraformaldehyde instillation, and removed for determination of lung wet/dry weight ratio.

\section{Statistics}

Data are presented as means \pm SD. Statistical analyses of physiological parameters were carried out by one-way ANOVA with Bonferroni tests for pairwise comparisons between each time-point and uninjured controls (represented as day 0). We used Spearman's correlation coefficients to test the correlations between RAGE levels and other continuous variables. Statistical significance was defined as $\mathrm{p}<0.05$.

\section{RESULTS}

\section{Physiology}

Mice showed significant weight loss in the first 3 days after acid instillation despite initial fluid resuscitation, which subsequently returned to pre-injury levels by day 10 (fig. 1a). During the first 2 days, animals showed reduced movement, piloerection and laboured breathing suggesting injury. Consistent with this, compared with uninjured animals (day 0), arterial oxygenation significantly deteriorated by day 1 after injury with gradual improvement by day 5 (fig. 1b). Mean arterial oxygen tension $\left(\left(\mathrm{Pa}_{\mathrm{a}} \mathrm{O}_{2}\right) / \mathrm{FI}_{1} \mathrm{O}_{2}\right.$ ratios $)$ achieved clinical ARDS criteria $(<200)$ on day 1 and ALI criteria $(<300)$ on days 2 and 3 . Respiratory system elastance substantially increased at days 1 and 2 after injury, and then returned toward baseline from day 3 (fig. 1c). There were no significant changes in respiratory system resistance (data not shown). Additionally, wet/dry weight ratio was determined to assess lung water content, and this showed a significant $61 \%$ increase at day 1 , which declined towards normal limits by day 3 (fig. 1d).

\section{Alveolar inflammation}

BAL cytology showed a dramatic change in cellularity within the alveolar space following injury (fig. $2 a$ and b). Before instillation, the cellular content was virtually exclusively alveolar macrophages. Following acid instillation the proportion of neutrophils increased dramatically, with BAL neutrophil numbers showing a peak on day 2 followed by a significant drop by day 3 and effective absence by day 10. In addition, we found a gradual increase in lymphocyte proportion following aspiration and, by day 10 (once neutrophils had disappeared), they constituted up to $15 \%$ of the total cell population (see online supplementary material). The temporal profile of neutrophil alveolitis was mirrored by changes in BALF levels of mouse neutrophil chemokines MIP-2 and KC (fig. 2c and d) and the pro-inflammatory mediators TNF- $\alpha$ and IL-6 (fig. 2e and f).

\section{Alveolar epithelial dysfunction}

Alveolar-capillary barrier permeability as assessed by BALF protein showed a substantial increase at day 1 with return to normal levels by day 10 (fig. 3a). BALF levels of RAGE were also significantly upregulated at day 1 , which subsequently reduced and were near baseline by day 3 (fig. $3 b$ ). BALF 
soluble RAGE levels were found to inversely correlate with

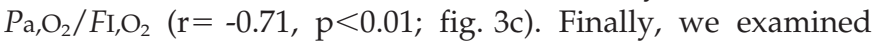
alveolar epithelial function through measurement of AFC rate (fig. 3d), which showed a significant deterioration during the first 2 days after acid instillation. Interestingly, the lungs regained the ability to clear fluid after day 3, suggesting a restoration of alveolar epithelial function.

\section{Lung injury scoring}

Acid aspiration caused substantial changes in lung architecture compared with uninjured controls. Over the first $48 \mathrm{~h}$ there were disrupted alveoli and the presence of fluid and haemorrhage
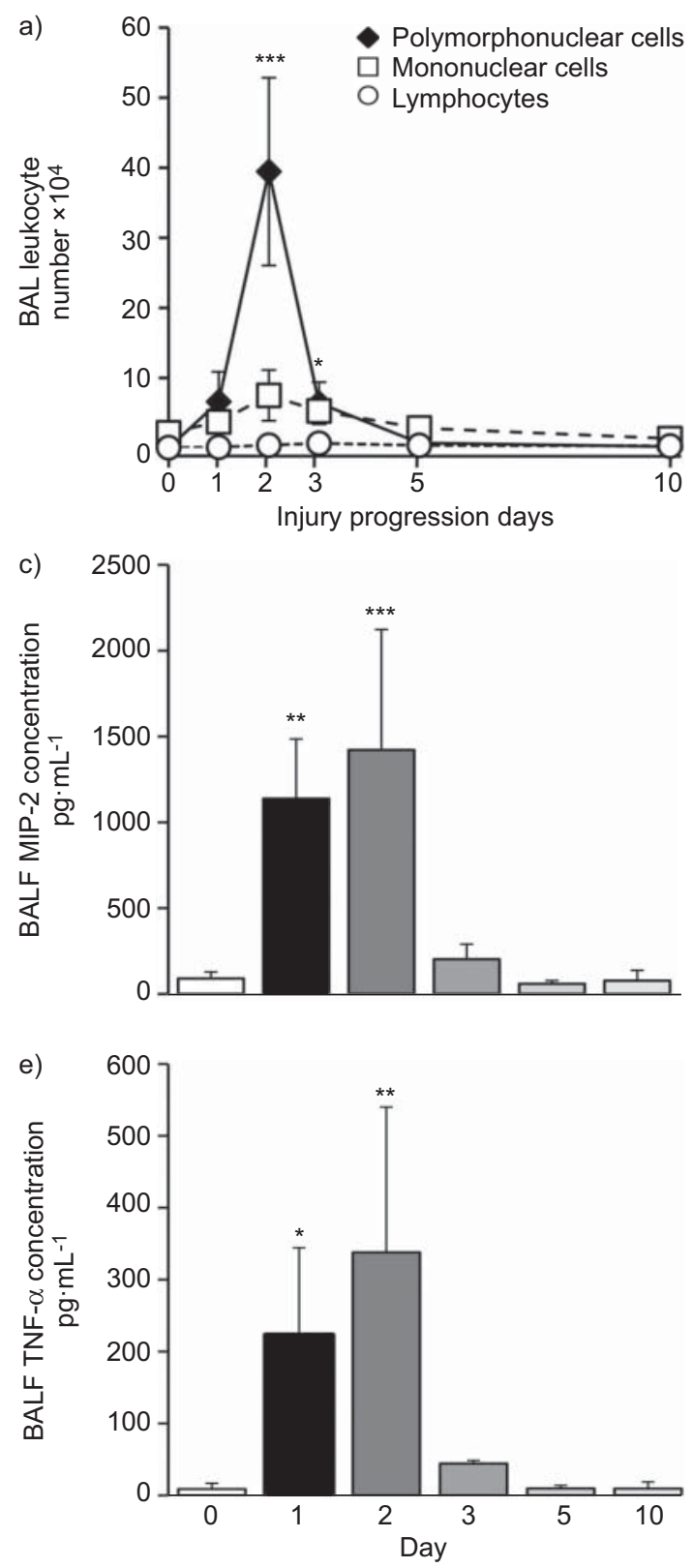

within the alveolar space (fig. 4a-c). The alveolar walls were markedly thickened with the presence of a neutrophilic and mononuclear infiltrate, and there was hyaline membrane deposition. After day 3 lung neutrophil infiltration, alveolar structure and debris were somewhat normalised, but there remained a cellular infiltrate, predominantly mononuclear in nature (fig. $4 \mathrm{~d}-\mathrm{f}$ ). Overall, lung injury scores were significantly increased on days 1 and 2 (fig. $4 \mathrm{~g}$ ). In addition, bronchiolar walls were thickened suggesting collagen deposition. Confirming this possibility, sections stained with Masson's trichrome showed increased collagen throughout, particularly in the walls of proximal to terminal bronchioles (fig. 5).
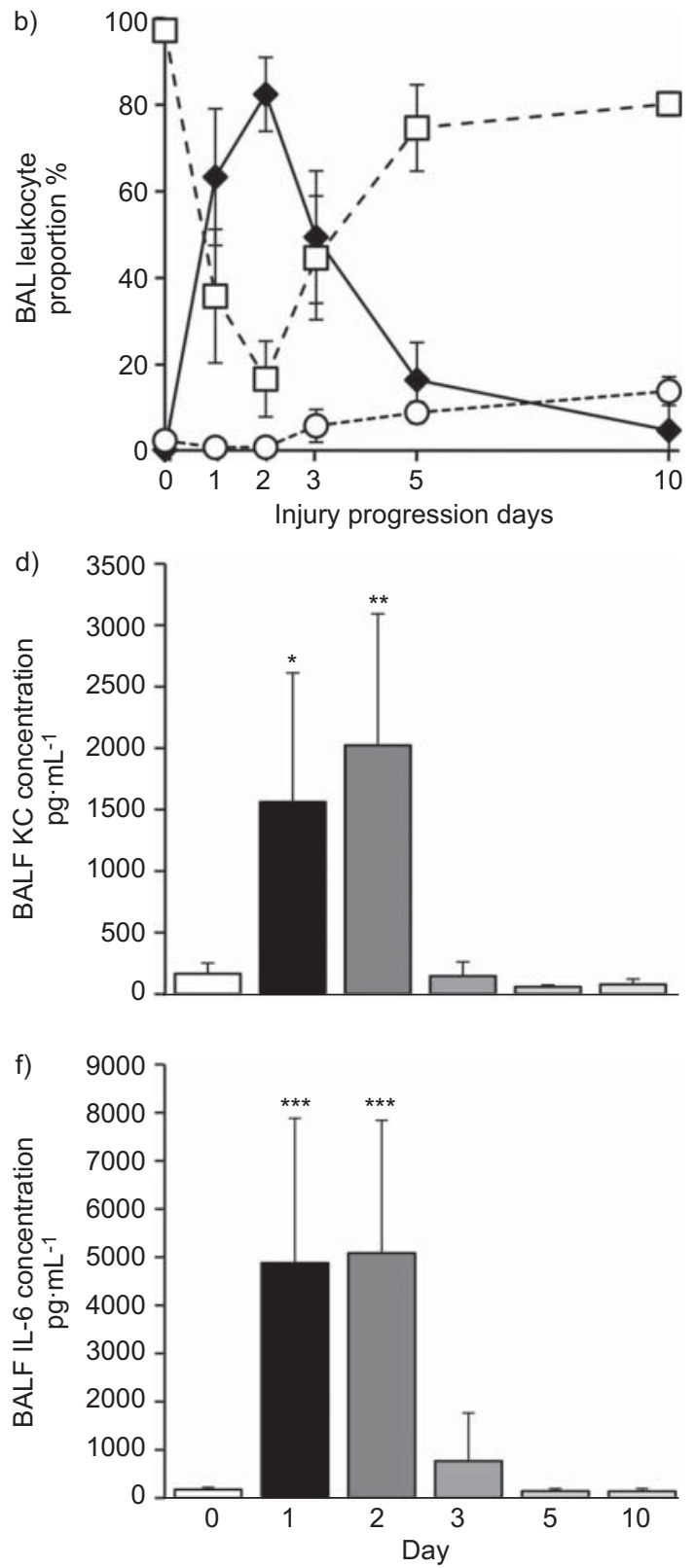

FIGURE 2. Bronchoalveolar lavage (BAL) a) numbers and b) proportions of polymorphonuclear neutrophils, mononuclear cells and lymphocytes within the model ( $\mathrm{n}=3-$ 5 for each time-point). Measurement of BAL fluid (BALF) levels of chemokines c) macrophage inflammatory protein (MIP)-2 and d) keratinocyte-derived chemokine (KC). BALF cytokines e) tumour necrosis factor (TNF)- $\alpha$ and $f$ ) interleukin (IL)-6 after acid aspiration ( $n=3-6$ for each time-point). *: $p<0.05$; **: $p<0.01 ; * \star *$ : $p<0.001$ versus day 0 uninjured controls. 

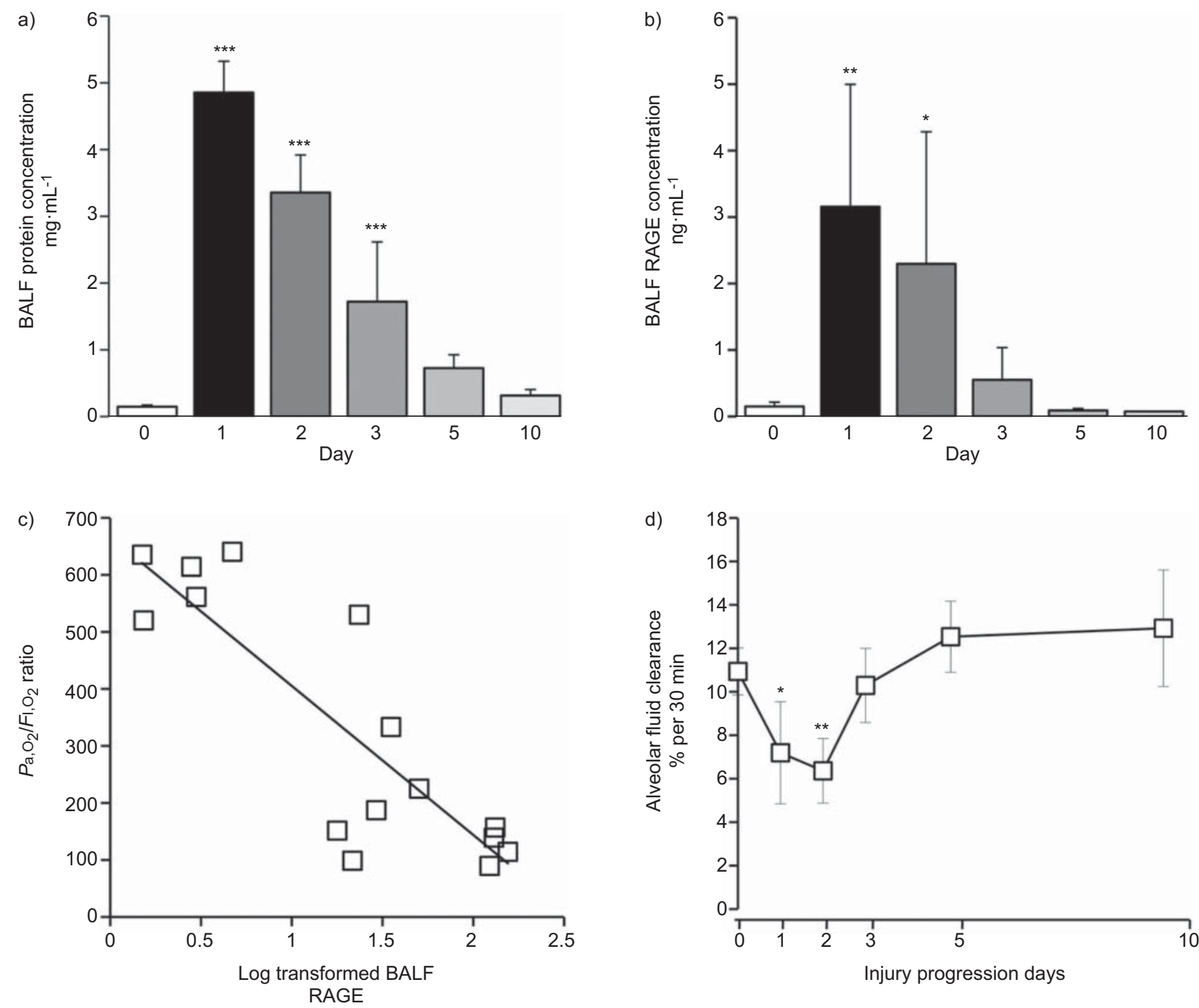

FIGURE 3. a) Changes in alveolar-capillary barrier permeability as measured by bronchoalveolar lavage fluid (BALF) protein level ( $n=3-6$ for each time-point) b) Measurement of BALF soluble receptor for advanced glycation end-products (RAGE) levels as a marker of type 1 alveolar epithelial injury ( $n=3-6$ for each time-point). c) Arterial oxygen tension $\left(\mathrm{Pa}_{2} \mathrm{O}_{2}\right)$ /inspiratory oxygen fraction $\left(\mathrm{Fl}_{1}, \mathrm{O}_{2}\right)$ plotted against log transformed RAGE shows that oxygenation inversely correlated with the level of alveolar RAGE $(r=-0.71, p<0.01)$. Each data-point represents a separate animal. $d)$ Measurement of alveolar fluid clearance rate as a marker of epithelial function $(n=3-6$ for each time-point). *: $p<0.05 ;{ }^{*}: p<0.01 ; * * *: p<0.001$ versus day 0 uninjured controls.

\section{DISCUSSION}

Although multiple potential molecular targets have been identified and shown to be effective in animal models of ALI, none has yet translated to clinical benefit for patients with ALI/ ARDS. One of the major pitfalls to translation from bench to bedside is the diversity of animal models that do not fully reproduce human conditions. Indeed, recent reviews have highlighted the features required to optimally model ALI/ ARDS, and that no current animal model replicates the complexities of this devastating disorder $[3,4,15]$. In this study, we describe a mouse model of ALI/ARDS induced by bilateral instillation of acid, which we believe represents a significant step forward in terms of reproducing the vast majority of the main pathophysiological hallmarks of clinical ALI/ARDS.

The primary goal of the current study was to develop a model that induced substantial injury but had low mortality over time, allowing investigation into the resolution/repair processes from this highly injured baseline. Existing models are rarely able to capture both of these aspects. Experimental paradigms, such as high-stretch mechanical ventilation [16], intravenous oleic acid [17], and sepsis induced by caecal ligation and puncture [18], can induce overwhelming injury over a timescale from hours to days, but do not then allow investigation into repair/resolution. Conversely, models such as intratracheal endotoxin and bleomycin may allow investigation of certain aspects surrounding resolution of inflammation and fibrotic processes that can occur following clinical ALI/ ARDS, but do not produce the substantial hypoxaemia that is a prerequisite for disease diagnosis [4].

Bilateral instillation of acid of similar concentrations to that used in this study has been reported to produce significant early mortality in mice [9]. This is most likely to be due to substantial 

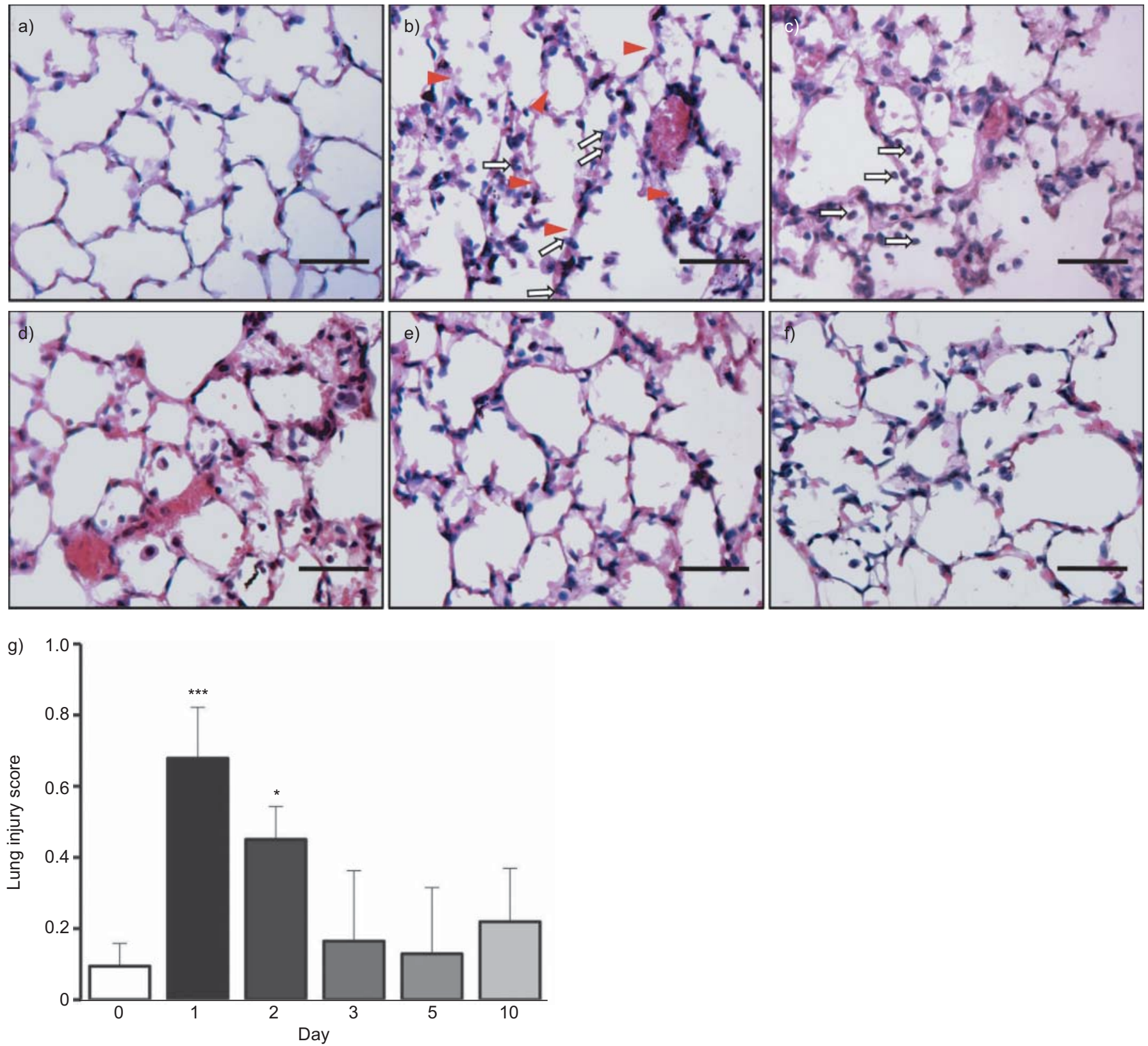

FIGURE 4. a-f) Representative haematoxylin and eosin (H\&E)-stained sections at $\times 400$ original magnification of uninjured and injured animals at all time-points after acid aspiration. a) Day 0, b) day 1, c) day 2, d) day 3, e) day 5 and f) day 10. There is greater cellularity consisting mainly of neutrophils (white arrows) on days 1 and 2 with more areas of atelectasis as well as increased alveolar disruption with hyaline membranes (arrowheads), proteinaceous debris and haemorrhage. With increasing time there is a reduction in cellularity as well as a shift from a neutrophilic to a predominantly mononuclear infiltrate. Scale bars $=50 \mu \mathrm{m}$. g) Lung injury scoring shows a significant injury on days 1 and 2 with a reduction from day 3 onwards ( $n=3-4$ for each time-point). Lung injury was assessed on a scale of $0-2$ for each of the following criteria: i) neutrophils in the alveolar space, ii) neutrophils in the interstitial space, iii) number of hyaline membranes, iv) amount of proteinaceous debris, and v) extent of alveolar septal thickening. The final injury score was derived from the following calculation: Score $=[20 \times$ (i) $+14 \times($ ii) $+7 \times$ (iii) $+7 \times$ (iv) $+2 \times(v)] /($ number of fields $\times 100)$. *: $p<0.05$; ***: $p<0.001$ versus day 0 uninjured controls.

bronchospasm and upper airway oedema caused by acid administration, and/or severe hypoxaemia during the acute phase of lung injury combined with respiratory depression produced by anaesthesia. Whereas other studies have circumvented this by reducing the dose/strength of acid $[8,9]$ or utilising mechanical ventilation [6], we found that careful airway manipulation during instillation, as well as the use of supplemental oxygen with maintenance of humidity/temperature over the initial hours after instillation successfully reduced mortality to zero. Acid instillation did, however, lead to a significant weight loss, which lasted for the first 3 days of injury, despite the initial fluid bolus given. We previously found, using exactly the same 

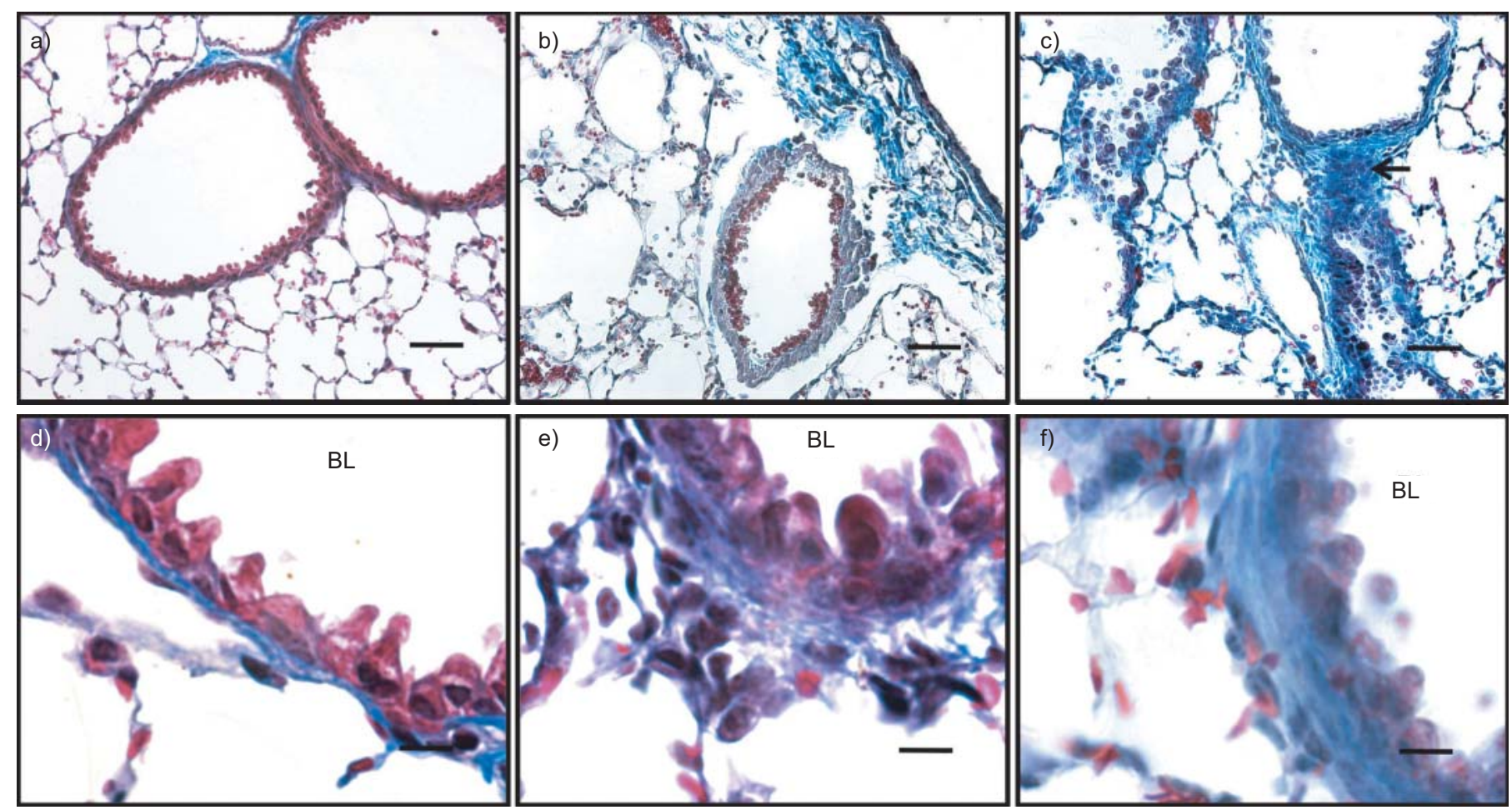

FIGURE 5. Representative Masson's trichrome stained sections at a-c) $\times 200$ (scale bars $=50 \mu \mathrm{m}$ ) and $\mathrm{d}-\mathrm{f}$ ) $\times 1000$ (scale bars $=10 \mu \mathrm{m})$ original magnification of uninjured ( $a$ and $d$ ), and injured animals at days $2(b$ and e) and 10 ( $c$ and f) after acid aspiration. There are significant changes, with cellular infiltration focussed in the peribronchiolar regions. Higher magnification images show increasing thickness of bronchiolar walls as a result of increased collagen deposition already at day 2 (e), and increasing over the course of the injury. This is particularly apparent at day 10 (c) which shows multiple fibrotic foci (black arrow). BL: bronchiolar lumen.

anaesthetic regimen and instillation technique, that low-dose endotoxin instillation leads to a weight loss of only $\sim 2 \%$ at $24 \mathrm{~h}$ [10], as compared with $\sim 8 \%$ in the present study. Thus, the degree of weight loss in the current model probably truly reflects disease severity and progression.

Due to their compliant rib cage, anaesthesia and surgical preparation in mice inevitably induces some alveolar collapse until mechanical ventilation is established. The degree of such lung collapse and its effects on respiratory physiology parameters are often difficult to control. We therefore assessed arterial blood gases and respiratory mechanics in these acidinjured animals (and untreated controls) after the rigorous standardisation of volume history of their lungs, i.e. a recruitment manoeuvre followed by a short period of positive pressure ventilation with $100 \% \quad \mathrm{O}_{2}$. During the first 3 days after aspiration, both respiratory system mechanics and arterial oxygenation were substantially deteriorated compared with normal. Indeed, oxygenation reached the criteria for clinical ALI/ARDS over the first 3 days, making the zero mortality and effectively full recovery by days 5-10 all the more remarkable. This lack of mortality during the peak of injury (days 1-2) may also be specific to the acid model with minimal haemodynamic compromise, as compared with other ALI models, such as highdose lipopolysaccharide, caecal ligation puncture and oleic acid.

Previous studies have demonstrated the importance of pulmonary inflammation within ALI. Animal studies have identified multiple inflammatory mediators that, when interfered with, attenuate injury [19], while clinical studies have shown strong correlations between various mediators and mortality [20]. Successful recovery from ALI therefore requires adequate and timely resolution of inflammation [21]. Although the majority of pre-clinical trials target inflammatory pathways prophylactically or very soon after injury induction, in reality most ALI patients present at a much later point in the progression of disease, implying that a more realistic target may be the enhancement of inflammatory resolution. The removal of neutrophils from the alveolar space is an absolute necessity for resolution to occur [22]. In the current model we found a peak of BAL neutrophil numbers at day 2 that dramatically declined by day 3 . The continued increase in neutrophils (and TNF- $\alpha$ levels) in the alveolar space up to $48 \mathrm{~h}$ is consistent with a previous study of acid aspiration [23], although that study did not investigate later time-points. The presence of neutrophils in the alveolar space and the interstitium are the major determinants of the lung injury score utilised in this study (see online supplementary material), and the reduced injury score from day 3 confirmed that neutrophils are removed not only from the alveoli but also the lung itself, rather than migrating/redistributing elsewhere within the lung compartments that is not accessible by BAL. Although the fate of neutrophils has not been specifically investigated, it is most likely that they undergo apoptosis followed by efferocytosis by alveolar macrophages [24, 25], which is crucial for resolution of the inflammatory response. The reduction in neutrophil levels was effectively mirrored by changes in soluble pro-inflammatory mediators, also consistent with a significant shift in the alveolar milieu from a proinflammatory state to a pro-resolution state between days 2-3. 
Finally, an increase in the proportion of lymphocytes within the alveolar space was apparent towards day 10 after injury, which may be consistent with recent work highlighting the potential involvement of regulatory T-cells in ALI resolution [26].

It has been shown that alveolar oedema formation in ALI/ARDS is associated with epithelial dysfunction [27]. The deterioration in respiratory mechanics observed reflects a combination of pulmonary oedema and alveolar collapse, and increased BALF protein levels and lung wet/dry weight ratio confirm significant alveolar permeability and oedema during the acute phase of injury in this model. A variety of specific biomarkers for epithelial injury during ALI have been proposed, with RAGE the most recently advocated [28]. RAGE is expressed on the basal surface of type 1 epithelial cells, and has been shown to increase in BALF and plasma of patients with ALI/ARDS [29]. In murine models of acid aspiration, the BALF RAGE level was also found to correlate strongly with the $\mathrm{pH}$ of acid instilled [30]. We found that BALF soluble RAGE levels correlate with oxygenation, which suggests that our model produces significant epithelial dysfunction and its subsequent resolution. In addition to worsening barrier permeability, we found that acid instillation led to reduced alveolar fluid clearance. Acid aspiration models have been shown to reduce AFC [31], but we show for the first time in this model an improvement in AFC after day 2, which is strongly suggestive of epithelial resolution and repair. This improvement coincides with the resolution of many other parameters on day 3 .

It is uncertain why ALI/ARDS resolution involves fibrosis in some patients but not others. Very few animal models allow investigation of this reparative process, particularly from a starting point of substantial initial injury. Evidence of increased collagen deposition from proximal to distal bronchioles was found within $48 \mathrm{~h}$ after acid administration, progressing to substantially increased numbers of fibrotic foci by day 10. It is possible that this fibrosis may explain why lung elastance did not completely revert to normal values at day 10 , maintaining a $16 \%$ increase compared with uninjured controls. Despite the generalised alveolar disruption observed on day 2, there was some return to recognisable alveolar architecture by day 10 and a reduction in alveolar debris. Although interstitial cellularity was still present at day 10, this mainly consisted of mononuclear cells and lymphocytes. Investigation of the relevance of such cell types (including monocyte and macrophage subsets) will be a major goal for further study utilising this model.

In conclusion, the acute injurious phase of the acid aspiration model has been well characterised and has been described as the most clinically applicable model available [32]. While previous studies utilising this model have frequently reduced (in our opinion) its translatability by using unilateral instillation or small doses of acid to limit injury, by careful airway management and recovery, we were able to develop a model that replicates major hallmarks of human ALI/ARDS, i.e. acute onset, bilateral injury leading to severe hypoxaemia with increased respiratory elastance, impaired alveolar fluid clearance, epithelial damage and barrier permeability, with repair involving fibrosis. While the degree of injury is difficult to compare with the human situation, the level of hypoxaemia is similar to that observed during clinical ALI/ARDS, and other parameters determined (BALF protein and lung wet/dry weight ratio) are of similar or greater magnitude to other models of "substantial/lethal" ALI (e.g. caecal ligation and puncture [33], oleic acid [34] and ventilator-induced lung injury [12]). We believe that this animal model provides a powerful translational platform to truly model clinically relevant situations by recapitulating human ALI/ARDS in terms of disease profile, severity and time course. Such a model should allow us to better investigate the pathological processes, the effects of clinically applicable secondary insults and the potential therapeutic interventions, particularly those focusing on resolution and repair.

\section{SUPPORT STATEMENT}

This work was supported by the Wellcome Trust (grants 081208 and 092851).

\section{STATEMENT OF INTEREST}

None declared.

\section{REFERENCES}

1 Rubenfeld GD, Caldwell E, Peabody E, et al. Incidence and outcomes of acute lung injury. N Engl J Med 2005; 353: 1685-1693.

2 Ware LB, Matthay MA. The acute respiratory distress syndrome. N Engl J Med 2000; 342: 1334-1349.

3 Matute-Bello G, Downey G, Moore BB, et al. An official American Thoracic Society Workshop report: features and measurements of experimental acute lung injury in animals. Am J Respir Cell Mol Biol 2011; 44: 725-738.

4 Matute-Bello G, Frevert CW, Martin TR. Animal models of acute lung injury. Am J Physiol Lung Cell Mol Physiol 2008; 295: L379-L399.

5 Ware LB. Modeling human lung disease in animals. Am J Physiol Lung Cell Mol Physiol 2008; 294: L149-L150.

6 Imai Y, Kuba K, Neely GG, et al. Identification of oxidative stress and Toll-like receptor 4 signaling as a key pathway of acute lung injury. Cell 2008; 133: 235-249.

7 Nagase T, Uozumi N, Ishii S, et al. Acute lung injury by sepsis and acid aspiration: a key role for cytosolic phospholipase A2. Nat Immunol 2000; 1: 42-46.

8 Amigoni M, Bellani G, Scanziani M, et al. Lung injury and recovery in a murine model of unilateral acid aspiration: functional, biochemical, and morphologic characterization. Anesthesiology 2008; 108: 1037-1046.

9 Fukunaga K, Kohli P, Bonnans C, et al. Cyclooxygenase 2 plays a pivotal role in the resolution of acute lung injury. J Immunol 2005; 174: 5033-5039.

10 Wilson MR, O'Dea KP, Dorr AD, et al. Efficacy and safety of inhaled carbon monoxide during pulmonary inflammation in mice. PLOS ONE 2010; 5: e11565.

11 Wilson MR, Choudhury S, Goddard ME, et al. High tidal volume upregulates intrapulmonary cytokines in an in vivo mouse model of ventilator-induced lung injury. J Appl Physiol 2003; 95: 1385-1393.

12 Wilson MR, Goddard ME, O'Dea KP, et al. Differential roles of p55 and p75 tumor necrosis factor receptors on stretch-induced pulmonary edema in mice. Am J Physiol Lung Cell Mol Physiol 2007; 293: L60-L68.

13 Ewart S, Levitt R, Mitzner W. Respiratory system mechanics in mice measured by end-inflation occlusion. J Appl Physiol 1995; 79: 560-566.

14 Garat C, Carter EP, Matthay MA. New in situ mouse model to quantify alveolar epithelial fluid clearance. J Appl Physiol 1998; 84: 1763-1767.

15 Bastarache JA, Blackwell TS. Development of animal models for the acute respiratory distress syndrome. Dis Model Mech 2009; 2: 218-223.

16 Wilson MR, O'Dea KP, Zhang D, et al. Role of lung-marginated monocytes in an in vivo mouse model of ventilator-induced lung injury. Am J Respir Crit Care Med 2009; 179: 914-922. 
17 Zhou Z, Kozlowski J, Schuster DP. Physiologic, biochemical, and imaging characterization of acute lung injury in mice. Am J Respir Crit Care Med 2005; 172: 344-351.

18 Toya SP, Li F, Bonini MG, et al. Interaction of a specific population of human embryonic stem cell-derived progenitor cells with $\mathrm{CD} 11 \mathrm{~b}+$ cells ameliorates sepsis-induced lung inflammatory injury. Am J Pathol 2011; 178: 313-324.

19 Uhlig S, Uhlig U. Pharmacological interventions in ventilatorinduced lung injury. Trends Pharmacol Sci 2004; 25: 592-600.

20 Ranieri VM, Suter PM, Tortorella C, et al. Effect of mechanical ventilation on inflammatory mediators in patients with acute respiratory distress syndrome: a randomized controlled trial. JAMA 1999; 282: 54-61.

21 Serhan CN., Ward PA., Gilroy DW. Fundamentals of Inflammation. Cambridge, Cambridge University Press, 2010; pp. 17-27.

22 Serhan CN, Savill J. Resolution of inflammation: the beginning programs the end. Nat Immunol 2005; 6: 1191-1197.

23 Segal BH, Davidson BA, Hutson AD, et al. Acid aspirationinduced lung inflammation and injury are exacerbated in NADPH oxidase-deficient mice. Am J Physiol Lung Cell Mol Physiol 2007; 292: L760-L768.

24 Hussain N, Wu F, Zhu L, et al. Neutrophil apoptosis during the development and resolution of oleic acid-induced acute lung injury in the rat. Am J Respir Cell Mol Biol 1998; 19: 867-874.

25 Cox G, Crossley J, Xing Z. Macrophage engulfment of apoptotic neutrophils contributes to the resolution of acute pulmonary inflammation in vivo. Am J Respir Cell Mol Biol 1995; 12: 232-237.
26 D'Alessio F, Tsushima K, Aggarwal N, et al. CD4+CD25+Foxp3+ Tregs resolve experimental lung injury in mice and are present in humans with acute lung injury. J Clin Invest 2009; 119: 2898-2913.

27 Matthay MA, Wiener-Kronish JP. Intact epithelial barrier function is critical for the resolution of alveolar edema in humans. Am Rev Respir Dis 1990; 142: 1250-1257.

28 Griffiths MJ, McAuley DF. RAGE: a biomarker for acute lung injury. Thorax 2008; 63: 1034-1036.

29 Briot R, Frank JA, Uchida T, et al. Elevated levels of the receptor for advanced glycation end products, a marker of alveolar epithelial type I cell injury, predict impaired alveolar fluid clearance in isolated perfused human lungs. Chest 2009; 135: 269-275.

30 Uchida T, Shirasawa M, Ware LB, et al. Receptor for advanced glycation end-products is a marker of type I cell injury in acute lung injury. Am J Respir Crit Care Med 2006; 173: 1008-1015.

31 Modelska K, Pittet JF, Folkesson HG, et al. Acid-induced lung injury. Protective effect of anti-interleukin- 8 pretreatment on alveolar epithelial barrier function in rabbits. Am J Respir Crit Care Med 1999; 160: 1450-1456.

32 Henzler D, Hochhausen N, Chankalal R, et al. Physiologic and biologic characteristics of three experimental models of acute lung injury in rats. Anesth Analg 2011; 112: 1139-1146.

33 Shen L, Mo H, Cai L, et al. Losartan prevents sepsis-induced acute lung injury and decreases activation of nuclear factor $\mathrm{\kappa B}$ and mitogen-activated protein kinases. Shock 2009; 31: 500-506.

34 Ulrich K, Stern M, Goddard ME, et al. Keratinocyte growth factor therapy in murine oleic acid-induced acute lung injury. Am J Physiol Lung Cell Mol Physiol 2005; 288: L1179-L1192. 